\title{
Brans-Dicke analogue of the Roberts geometry
}

\author{
Bardia H. Fahim, , 因 Valerio Faraoni, ${ }^{1,0}$ and Andrea Giusti ${ }^{1}$, 团 \\ ${ }^{1}$ Department of Physics \& Astronomy, Bishop's University, \\ 2600 College Street, Sherbrooke, Québec, Canada J1M 1Z77
}

\begin{abstract}
We report a new one-parameter family of spherically symmetric, inhomogeneous, and timedependent solutions of the vacuum Brans-Dicke field equations which are conformal to the Roberts scalar field geometries of Einstein gravity. The new solution is spherical and time-dependent and contains a naked central singularity. We use it as a seed to generate another two-parameter family of solutions using a known symmetry of vacuum Brans-Dicke gravity.
\end{abstract}

\section{INTRODUCTION}

Einstein's theory of General Relativity (GR) conflicts with quantum mechanics, as demonstrated by the fact that attempts to quantize GR produce, in the low-energy limit, theories of gravity which deviate from GR due to extra degrees of freedom or higher order terms in their field equations. From the theoretical point of view, therefore, GR must be modified in order to make it compatible with quantum field theory. A more urgent motivation to explore gravity beyond Einstein theory comes from observational cosmology. If the present acceleration of the universe discovered in 1998 with type Ia supernovae is to be explained within the realm of GR, one needs an incredibly fine-tuned cosmological constant $\Lambda$ or a completely ad hoc dark energy sourcing the Einstein equations. Instead of postulating these rather embarassing and ad hoc ingredients of the standard $\Lambda$-Cold Dark Matter $(\Lambda \mathrm{CDM})$ cosmological model, a viable alternative consists of modifying gravity at large scales, while preserving GR at small scales. Moreover, while the linear approximation to GR is well tested at Solar System scales and has received a spectacular confirmation with the LIGO detections of gravitational waves [1 4 ], the theory is not tested at most spatial scales and in most curvature regimes [5, 6]. Currently, the most popular alternative to GR to explain the cosmic acceleration without invoking dark energy is probably the class of $f(\mathcal{R})$ theories of gravity, where $\mathcal{R}$ is the Ricci scalar of the metric connection [7] (see [8] for reviews). $f(\mathcal{R})$ theories are nothing but scalar-tensor theories in disguise with vanishing Brans-Dicke coupling $\omega$ and equipped with a complicated potential for the scalar degree of freedom $f^{\prime}(\mathcal{R})$ [8].

Brans-Dicke theory [9] is the prototypical alternative to GR and the simplest representative of scalar-tensor gravity [10]. Analytical solutions of the field equations are useful to understand the physics of this theory and of its scalar-tensor generalizations. Inhomogeneous and timedependent scalar field solutions are rare both in Einstein and in alternative gravity and it is interesting to expand the meagre catalogue available. One example of spheri- cally symmetric, inhomogeneous, and time-dependent solution of the Einstein equations is the Roberts geometry sourced by a massless, minimally coupled scalar field 1 12. The Roberts solution has been used as an example in the study of critical phenomena occurring during the gravitational collapse of scalar fields [13 -16], is of interest for Cosmic Censorship [12] and wormhole formation [1722], and has been the subject of some attention due to the difficulty of obtaining dynamical and inhomogeneous analytical solutions of the Einstein equations [20, 21, 23].

The Roberts geometry [12] (with an error corrected in Refs. [14, 15, 23 26]) is a spherical, continuously selfsimilar spacetime since it admits a homothetic Killing vector field $\xi^{c}$ satisfying $£_{\xi} g_{a b}=2 \lambda g_{a b}$, where $\lambda$ is a constant 12]. Its stability was studied in [27, 28]. Here we adopt 2 the corrected and slightly generalized Roberts solution given by Burko in double null coordinates $(u, v, \vartheta, \varphi)[23]$,

$$
\begin{gathered}
d \tilde{s}^{2}=-d u d v+r^{2}(u, v) d \Omega_{(2)}^{2}, \\
r^{2}(u, v)=\frac{1}{4}\left[\left(1-4 \sigma^{2}\right) v^{2}-2 u v+u^{2}\right],
\end{gathered}
$$

where $d \Omega_{(2)}^{2} \equiv d \vartheta^{2}+\sin ^{2} \vartheta d \varphi^{2}$ is the line element on the unit 2-sphere and the scalar field is

$$
\tilde{\phi}= \pm \frac{1}{2} \ln \left[\frac{(1-2 \sigma) v-u}{(1+2 \sigma) v-u}\right]
$$

where $\sigma$ is a constant. In order for the areal radius squared (1.2) and for the argument of the logarithm in Eq. (1.3) to be positive, it must be $|\sigma|<1 / 2$. In the limit $\sigma \rightarrow 0$ the scalar $\tilde{\phi}$ disappears and one recovers Minkowski spacetime. We also note that the right hand side of Eq. (1.2) can be written as

$$
r^{2}(u, v)=\frac{1}{4}[(1-2 \sigma) v-u][(1+2 \sigma) v-u],
$$

which will be useful later. Therefore, the origin $r=0$ corresponds to $u=(1 \pm 2 \sigma) v$. Since $r^{2} \geq 0$, Eq. (1.4)

\footnotetext{
${ }^{1}$ A version with a conformally coupled scalar has also been proposed [11].

${ }^{2}$ We follow the notations of Ref. [29].
}

\footnotetext{
* bardia.fahim@usask.ca

† vfaraoni@ubishops.ca

$\ddagger$ agiusti@ubishops.ca
} 
guarantees that the argument of the logarithm in the Roberts scalar field (1.3) is positive (the absolute value of this argument appearing in this equation in [23] is redundant). In the region in which the gradient of the scalar field is timelike, the Roberts solution reproduces 23. a 1967 stiff fluid solution of Gutman and Bespalko [30].

The Einstein equations reduce to

$$
\tilde{R}_{a b}=8 \pi \nabla_{a} \tilde{\phi} \nabla_{b} \tilde{\phi}
$$

where $\tilde{R}_{a b}$ is the Ricci tensor, while the Ricci scalar is

$$
\tilde{R}=8 \pi \tilde{g}^{a b} \nabla_{a} \tilde{\phi} \nabla_{b} \tilde{\phi}=-32 \pi \tilde{\phi}_{, u} \tilde{\phi}_{, v}=\frac{8 \pi \sigma^{2} u v}{r^{4}}
$$

and diverges as $r \rightarrow 0$, where there is a spacetime singularity. This singularity is not covered by apparent horizons, whose radii would be the positive roots of the equation $\tilde{g}^{a b} \nabla_{a} r \nabla_{b} r=0$. For the Roberts solution, this equation reads

$$
\tilde{g}^{a b} \nabla_{a} r \nabla_{b} r=-\frac{1}{4 r^{2}}\left[\left(1-4 \sigma^{2}\right) v-u\right](u-v)=0
$$

and the only roots are $v=u$ or $v=\frac{u}{1-4 \sigma^{2}}$ (remember that $|\sigma|<1 / 2$ ). But, using Eq. (1.2), $v=u$ would imply $r^{2}=-\sigma^{2} u^{2}<0$, which is impossible. Similarly, $v=u /\left(1-4 \sigma^{2}\right)$ would imply $r^{2}=-\frac{\sigma^{2} u^{2}}{1-4 \sigma^{2}}<0$, which is also impossible (the exceptions are $u=v=0$ which corresponds to $r=0$ and to the absence of apparent horizon positive roots). Therefore, there are no apparent horizons and the central singularity is naked. The Misner-Sharp-Hernandez mass $M_{\mathrm{MSH}}$ is defined by $1-2 M_{\mathrm{MSH}} / r=\tilde{g}^{a b} \nabla_{a} r \nabla_{b} r$ [31, 32], which gives

$$
M_{\mathrm{MSH}}=\frac{r}{2}\left(1-\tilde{g}^{a b} \nabla_{a} r \nabla_{b} r\right)=-\frac{\sigma^{2} u v}{2 r} .
$$

It is negative in the entire region $u v>0$ forming the past and future light cone of the origin $r=0$.

In the following, we regard the Roberts scalar field solution of GR as the Einstein frame version of a BransDicke counterpart, which constitutes a new solution explored in this paper. Analytical solutions of scalar-tensor gravity with the gravitational scalar field $\phi$ propagating along null fronts are unknown, except for certain exact plane waves - some of them are obtained by matching spacetime regions along null shells [33, 34] and others are exotic stealth wave solutions of Brans-Dicke-Maxwell gravity 35]. It is interesting, therefore, to examine the Brans-Dicke analogue of the Roberts solution and, possibly, similar solutions of $f(\mathcal{R})$ gravity.

Let us recall some basics: the Jordan frame action of vacuum Brans-Dicke theory is [9]

$$
S_{\mathrm{BD}}=\int d^{4} x \frac{\sqrt{-g}}{16 \pi}\left(\phi \mathcal{R}-\frac{\omega}{\phi} \nabla^{c} \phi \nabla_{c} \phi-V(\phi)\right),
$$

where $\phi$ is the gravitational scalar field (approximately equivalent to the inverse of the gravitational coupling
$\left.G_{\text {eff }}\right), V(\phi)$ is the scalar field potential, and $\omega$ is the constant "Brans-Dicke coupling" parameter, while $g$ is the determinant of the spacetime metric $g_{a b}$. By varying this action, one obtains the vacuum Brans-Dicke field equations [9]

$$
\begin{aligned}
R_{a b}-\frac{\mathcal{R}}{2} g_{a b}= & \frac{\omega}{\phi^{2}}\left(\nabla_{a} \phi \nabla_{b} \phi-\frac{1}{2} g_{a b} \nabla^{c} \phi \nabla_{c} \phi\right) \\
& +\frac{1}{\phi}\left(\nabla_{a} \nabla_{b} \phi-g_{a b} \square \phi\right)-\frac{V}{2 \phi} g_{a b} \\
\square \phi= & \frac{1}{2 \omega+3}\left(\phi \frac{d V}{d \phi}-2 V\right) .
\end{aligned}
$$

A second representation of scalar-tensor gravity, the Einstein conformal frame [36] is obtained by means of the conformal transformation of the metric

$$
g_{a b} \rightarrow \tilde{g}_{a b}=\phi g_{a b}
$$

and the scalar field redefinition

$$
\phi \rightarrow \tilde{\phi}=\sqrt{\frac{|2 \omega+3|}{16 \pi}} \ln \left(\frac{\phi}{\phi_{*}}\right)
$$

where $\phi_{*}$ is a constant and $\omega \neq-3 / 2$. In terms of these new variables, the vacuum Brans-Dicke action (1.9) assumes the Einstein frame form

$$
S_{\mathrm{BD}}=\int d^{4} x \sqrt{-\tilde{g}}\left[\frac{\tilde{\mathcal{R}}}{16 \pi}-\frac{1}{2} \tilde{g}^{a b} \nabla_{a} \tilde{\phi} \nabla_{b} \tilde{\phi}-\tilde{V}(\tilde{\phi})\right]
$$

where

$$
\tilde{V}(\tilde{\phi})=\left.\frac{V(\phi)}{\phi^{2}}\right|_{\phi=\phi(\tilde{\phi})}
$$

We label Einstein frame quantities with a tilde. Formally, (1.14) is the Einstein-Hilbert action coupled to a standard matter scalar field. The vacuum field equations in the Einstein frame are

$$
\begin{aligned}
& \tilde{R}_{a b}-\frac{1}{2} \tilde{g}_{a b} \tilde{\mathcal{R}}= 8 \pi\left(\nabla_{a} \tilde{\phi} \nabla_{b} \tilde{\phi}-\frac{1}{2} \tilde{g}_{a b} \tilde{g}^{c d} \nabla_{c} \tilde{\phi} \nabla_{d} \tilde{\phi}\right) \\
&-\tilde{V}(\tilde{\phi}) \tilde{g}_{a b} \\
& \tilde{g}^{a b} \tilde{\nabla}_{a} \tilde{\nabla}_{b} \tilde{\phi}-\frac{d \tilde{V}}{d \tilde{\phi}}=0
\end{aligned}
$$

Given a solution of the Einstein equations sourced by a minimally coupled scalar field, we can interpret it as the Einstein frame counterpart of a Jordan frame BransDicke gravity and map it to its representation in the Jordan frame. As a caveat, in general the Jordan frame scalar field potential $V(\phi)$ obtained from a reasonable Einstein frame potential $\tilde{V}(\tilde{\phi})$ is physically unmotivated, but this fact will not be of concern here since the Roberts 
solution that we consider has zero potential and this property transfers to the Jordan frame, as is well known [37, 38].

Now, on to $f(\mathcal{R})$ gravity: metric $f(\mathcal{R})$ theories of gravity are a subclass of Brans-Dicke gravity with action [8]

$$
S=\int d^{4} x \frac{\sqrt{-g}}{16 \pi} f(\mathcal{R})
$$

in vacuo, where $f(\mathcal{R})$ is a non-linear function of the Ricci scalar $\mathcal{R}$. By introducing the new scalar field $\phi \equiv f^{\prime}(\mathcal{R})$ with potential

$$
V(\phi)=\phi \mathcal{R}(\phi)-f(\mathcal{R}(\phi)),
$$

one shows [8] that the action (1.18) is equivalent to the vacuum Brans-Dicke action

$$
S=\int d^{4} x \frac{\sqrt{-g}}{16 \pi}[\phi \mathcal{R}-V(\phi)],
$$

with Brans-Dicke parameter $\omega=0$ and the potential (1.19).

\section{A NEW SOLUTION OF VACUUM BRANS-DICKE THEORY}

We now regard the Roberts spacetime $\left(\tilde{g}_{a b}, \tilde{\phi}\right)$ as the Einstein frame version of a Brans-Dicke solution $\left(g_{a b}, \phi\right)$, which we map back to the Jordan conformal frame. Their relation is

$$
\begin{gathered}
d s^{2}=\phi^{-1} d \tilde{s}^{2}, \\
\phi=\phi_{*} \exp \left[\sqrt{\frac{16 \pi}{|2 \omega+3|}} \tilde{\phi}\right] .
\end{gathered}
$$

Applying these on the Roberts solution (1.1)-(1.3) yields

$$
\begin{gathered}
d s^{2}=\left[\frac{(1-2 \sigma) v-u}{(1+2 \sigma) v-u}\right]^{\mp 2 \sqrt{\frac{\pi}{\mid 2 \omega+3}}}\left[-d u d v+r^{2}(u, v) d \Omega_{(2)}^{2}\right] \\
\phi=\phi_{*}\left[\frac{(1-2 \sigma) v-u}{(1+2 \sigma) v-u}\right]^{ \pm 2 \sqrt{\frac{\pi}{|2 \omega+3|}}}
\end{gathered}
$$

where $\phi_{*}>0$ (this constant is omitted without consequences in the expression of $d s^{2}$ ) and $r$ is given by Eq. (1.2).

The Jordan frame areal radius is

$$
\begin{aligned}
R(u, v)= & \frac{r(u, v)}{\sqrt{\phi}}=\frac{1}{2}\left[\frac{(1-2 \sigma) v-u}{(1+2 \sigma) v-u}\right]^{\mp \sqrt{\frac{\pi}{|2 \omega+3|}}} \\
& \times \sqrt{\left(1-4 \sigma^{2}\right) v^{2}-2 u v+u^{2}}
\end{aligned}
$$

where we used Eq. (1.2), from which it follows that the Jordan frame origin $R=0$ corresponds to $r=0$ or to $u=(1 \pm 2 \sigma) v$, which gives again $r=0$, therefore there is a one-to-one correspondence between $R=0$ and $r=$ 0 . The Jordan frame scalar (2.4) diverges at the origin $R=0$.

When they exist, the apparent horizons of a spherically symmetric metric are located by the roots of the equation $\nabla^{c} R \nabla_{c} R=0$, which here takes the form

$$
\begin{aligned}
\nabla^{c} R \nabla_{c} R & =2 g^{u v} R_{, u} R_{, v}=-4 \phi R_{, u} R_{, v} \\
& =-4\left(r_{, u}-\frac{r \phi, u}{2 \phi}\right)\left(r_{, v}-\frac{r \phi_{, v}}{2 \phi}\right),
\end{aligned}
$$

where we used the inverse metric

$$
\left(g^{\mu \nu}\right)=\left(\begin{array}{cccc}
0 & -2 \phi & 0 & 0 \\
-2 \phi & 0 & 0 & 0 \\
0 & 0 & \frac{\phi}{r^{2}} & 0 \\
0 & 0 & 0 & \frac{\phi}{r^{2} \sin ^{2} \vartheta}
\end{array}\right) .
$$

We must recover Minkowski space in the limit $\sigma \rightarrow 0$ in which $\phi \rightarrow$ const. and $d s^{2} \rightarrow-d u d v+r^{2}(u, v) d \Omega_{(2)}^{2}$. Then, $R$ depends on both $u$ and $v$ in an essential way and this is true also for $\sigma \neq 0$, hence the vanishing of $R_{, u}$ or $R_{, v}$ does not make sense physically. We conclude that there are no roots of Eq. (2.6) and no apparent horizons.

The Jordan frame Ricci scalar is obtained from the trace of Eq. 1.10

$$
\mathcal{R}=\frac{\omega}{\phi^{2}} \nabla^{a} \phi \nabla_{a} \phi+\frac{3 \square \phi}{\phi}+\frac{2 \tilde{V}}{\phi},
$$

where the second and third terms in the right hand side are zero, as $\phi$ is a free scalar field that satisfies $\square \phi=0$ (cf. Eq. (1.11)). The Ricci scalar becomes

$$
\begin{aligned}
\mathcal{R} & =\frac{\omega}{\phi^{2}} g^{a b} \nabla_{a} \phi \nabla_{b} \phi=\frac{2 \omega}{\phi^{2}} g^{u v} \partial_{u} \phi \partial_{v} \phi \\
& =\frac{16 \pi \omega \sigma^{2}}{|2 \omega+3|} \frac{\phi u v}{r^{4}}=\frac{16 \pi \omega \sigma^{2}}{|2 \omega+3|} \frac{u v}{\phi R^{4}},
\end{aligned}
$$

where we used the inverse metric (2.7) and

$$
\begin{aligned}
& \partial_{u} \phi=\mp 2 \sigma \sqrt{\frac{\pi}{|2 \omega+3|}} \frac{\phi v}{r^{2}}=\mp 2 \sigma \sqrt{\frac{\pi}{|2 \omega+3|}} \frac{v}{R^{2}}, \\
& \partial_{v} \phi= \pm 2 \sigma \sqrt{\frac{\pi}{|2 \omega+3|}} \frac{\phi u}{r^{2}}= \pm 2 \sigma \sqrt{\frac{\pi}{|2 \omega+3|}} \frac{u}{R^{2}} .
\end{aligned}
$$

When $\omega \neq 0$, the Ricci scalar diverges as $R \rightarrow 0$ together with $\phi$ and there exists a naked central singularity. When $\omega=0$, the Ricci scalar vanishes identically, but the Brans-Dicke scalar field $\phi$ still diverges at the origin with the Kretschmann scalar $\mathcal{K} \equiv R_{a b c d} R^{a b c d}$. This is given, for general values of $\omega$, by 


$$
\begin{aligned}
\mathcal{K}=\frac{4 \sigma^{2} \phi^{2}}{|2 \omega+3|^{2} r^{8}}\{ & \pm 4 \sqrt{\pi} \sigma u v|2 \omega+3|^{3 / 2}\left[\left(4 \sigma^{2}-1\right) v^{2}+u^{2}\right]+3 \sigma^{2} u^{2} v^{2}(2 \omega+3)^{2} \\
& +2 \pi|2 \omega+3|\left[2\left(10 \sigma^{2}-3\right) u^{2} v^{2}+2\left(1-4 \sigma^{2}\right) u v^{3}+\left(1-4 \sigma^{2}\right)^{2} v^{4}+2 u^{3} v+u^{4}\right] \\
& \left. \pm 16 \pi^{3 / 2} \sigma u v \sqrt{|2 \omega+3|}\left[\left(4 \sigma^{2}-1\right) v^{2}+u^{2}\right]+48 \pi^{2} \sigma^{2} u^{2} v^{2}\right\}
\end{aligned}
$$

\section{III. "CONFORMAL ROBERTS" IS NOT A SOLUTION OF $f(\mathcal{R})$ GRAVITY}

Let us consider now the possibility that the new BransDicke solution is also a solution of $f(\mathcal{R})$ gravity. Since there is no potential, it must be (cf. Eq. (1.19))

$$
V(\phi)=\phi \mathcal{R}-f(\mathcal{R})=0
$$

which integrates to $f(\mathcal{R})=f_{0} \mathcal{R}$, where $f_{0}$ is a constant. In addition, it must be $\omega=0$ and then the putative solution becomes

$$
d s_{(0)}^{2}=\left[\frac{(1-2 \sigma) v-u}{(1+2 \sigma) v-u}\right]^{\mp 2 \sqrt{\pi / 3}}\left[-d u d v+r^{2}(u, v) d \Omega_{(2)}^{2}\right]
$$

$$
\psi=\phi_{*}\left[\frac{(1-2 \sigma) v-u}{(1+2 \sigma) v-u}\right]^{ \pm 2 \sqrt{\pi / 3}}
$$

and it would seem that these expressions could provide a solution of GR. This is not true because, according to Eq. 2.9), $\omega=0$ also implies $\mathcal{R}=0$ and, in GR, it is instead $\mathcal{R} \propto \nabla^{c} \psi \nabla_{c} \psi$ which, in general, is incompatible with $\mathcal{R}=0$.

We conclude that the vacuum Brans-Dicke geometry (3.2), (1.2), and (3.3) is not a solution of $f(\mathcal{R})$ gravity nor of the Einstein-Klein-Gordon equations.

\section{ANOTHER TWO-PARAMETER FAMILY OF SOLUTIONS}

Vacuum Brans-Dicke theory is invariant under the operation [39]

$$
\begin{gathered}
g_{a b} \rightarrow \hat{g}_{a b}=\phi^{2 \alpha} g_{a b}, \\
\phi \rightarrow \hat{\phi}=\phi^{1-2 \alpha},
\end{gathered}
$$

for $\alpha \neq 1 / 2$ (the conformal transformation of the metric (4.1) has nothing to do with the conformal map relating Jordan and Einstein frames). A hat denotes geometric quantities constructed with the conformally rescaled metric $\hat{g}_{a b}$. The well-known transformation properties under the map $\hat{g}_{a b}=\Omega^{2} g_{a b}$ [29, 40, 42]

$$
\begin{aligned}
\hat{g}^{a b} & =\Omega^{-2} g^{a b}, \\
\sqrt{-\hat{g}} & =\Omega^{4} \sqrt{-g}, \\
\hat{R} & =\Omega^{-2}\left(R-\frac{6 \square \Omega}{\Omega}\right),
\end{aligned}
$$

plus the use of Eq. (4.2) yield

$$
\begin{aligned}
R= & \phi^{2 \alpha} \hat{R}-\frac{6 \alpha(1-\alpha)}{(1-2 \alpha)^{2}} \phi^{6 \alpha-2} \hat{g}^{a b} \hat{\nabla}_{a} \hat{\phi} \hat{\nabla}_{b} \hat{\phi} \\
& +\frac{6 \alpha}{1-2 \alpha} \phi^{4 \alpha-1} \hat{\square} \hat{\phi} .
\end{aligned}
$$

The d'Alembertian in the right hand side of Eq. (4.6) can be written as

$$
\frac{6 \alpha}{1-2 \alpha} \sqrt{-\hat{g}} \hat{\square} \hat{\phi}=\frac{6 \alpha}{1-2 \alpha} \partial_{\mu}\left(\sqrt{-\hat{g}} \hat{g}^{\mu \nu} \partial_{\nu} \hat{\phi}\right)
$$

and is integrated to a boundary term when placed in the action integral. By dropping this term (which is irrelevant in the variation leading to the Brans-Dicke field equations), the vacuum Brans-Dicke action (1.9) without potential reads

$$
\begin{aligned}
S_{\mathrm{BD}}= & \int d^{4} x \sqrt{-\hat{g}}\left\{\hat{\phi} \hat{R}-\left[\frac{\omega}{(1-2 \alpha)^{2}}+\frac{6 \alpha(1-\alpha)}{(1-2 \alpha)^{2}}\right]\right. \\
& \left.\frac{\hat{g}^{a b}}{\hat{\phi}} \hat{\nabla}_{a} \hat{\phi} \hat{\nabla}_{b} \hat{\phi}\right\} .
\end{aligned}
$$

If we rename the Brans-Dicke coupling as

$$
\hat{\omega}(\omega, \alpha)=\frac{\omega+6 \alpha(1-\alpha)}{(1-2 \alpha)^{2}}
$$

then the action reads 39

$$
S_{\mathrm{BD}}=\int d^{4} x \sqrt{-\hat{g}}\left[\hat{\phi} \hat{R}-\frac{\hat{\omega}}{\hat{\phi}} \hat{g}^{a b} \hat{\nabla}_{a} \hat{\phi} \hat{\nabla}_{b} \hat{\phi}\right]
$$

which is again of the Brans-Dicke form. Therefore, the operation (4.1), (4.2), and (4.9) is a symmetry of vacuum Brans-Dicke theory. It can be shown that, as $\alpha$ varies, it spans a one-parameter Abelian group of symmetries [39]. This property can be used to generate new solutions [43]. Here, using the conformal relative of the Roberts solution 
as a seed, we generate the new two-parameter family of solutions of vacuum Brans-Dicke gravity

$$
\begin{gathered}
d \hat{s}^{2}=\phi^{2 \alpha-1}\left[-d u d v+r^{2}(u, v) d \Omega_{(2)}^{2}\right], \\
\hat{\phi}=\phi_{0}\left[\frac{(1-2 \sigma) v-u}{(1+2 \sigma) v-u}\right]^{ \pm 2(1-2 \alpha) \sqrt{\frac{\pi}{2 \omega+3 \mid}}},
\end{gathered}
$$

with $r^{2}$ given by Eq. (1.2) and the Brans-Dicke coupling by Eq. (4.9). These new solutions, labelled by the parameters $\sigma$ and $\alpha$ (or, equivalently, $\sigma$ and $\hat{\omega}$ ) are spherically symmetric, time-dependent, and conformal to the Roberts spacetime. By repeating the analysis of the previous section, one concludes that there is again a central naked singularity where the scalar field diverges.

\section{CONCLUSIONS}

The geometry and Brans-Dicke scalar field (2.3), (1.2), and 2.4 constitute a new solution of vacuum Brans-Dicke theory without potential in the Jordan frame. By construction, this solution is conformal to the Roberts solution (1.1), (1.2), (1.3) of GR with a minimally coupled, massless scalar field as the matter source. This new solution has no apparent horizons and harbours a central naked singularity, where the Ricci curvature $\mathcal{R}$, the Kretschmann scalar $R_{a b c d} R^{a b c d}$, and the Brans-Dicke scalar $\phi$ diverge. This spacetime structure is the same as that of its Einstein frame cousin (studied in [12]) used to generate our new solution.

A generalization of the Roberts solution to an Antide Sitter "background", conformal to the geometry (1.1), (1.2) has been found by Roberts 44 and is in principle useful in studies of the AdS/CFT correspondence. This solution is conformal to the $\Lambda=0$ Roberts solution [21, 44]. We do not consider it here because, when mapped to the Jordan frame, the negative cosmological constant $\Lambda$ of the AdS background gives rise to the negative potential $V(\phi)=\Lambda \phi^{2} /(8 \pi)$ [45], as described by Eq. 1.15), and to an imaginary scalar field mass $\left(m^{2}=\frac{\Lambda}{8 \pi}\right)$ and is, therefore, of no physical interest.

The fact that the scalar field of the Roberts-AdS spacetime is exactly the same as in the Roberts solution without $\Lambda$ has been reported as interesting or surprising [21, 44]: in light of the fact that a cosmological constant in the Einstein frame corresponds to a mass term in the Jordan frame, and that a potential $V(\phi)=m^{2} \phi^{2} / 2$ disappears completely from the KleinGordon equation (1.11), this fact is perhaps not too surprising. Instead of mapping the Roberts-Anti-de Sitter geometry to the Jordan frame, we have used a known symmetry of vacuum Brans-Dicke theory to generate a new two-parameter family of solutions.

Scalar fields in various theories of gravity either collapse to GR black holes, as stated by well-known no-hair theorems [46], or they seem to generate only naked singularities or wormhole throats. Outside of the context of asymptotically flat and stationary GR or scalar-tensor gravity, a general theorem is not available. Future studies will explore this direction.

\section{ACKNOWLEDGMENTS}

This work is supported, in part, by the Natural Sciences \& Engineering Research Council of Canada (Grant no. 2016-03803 to V.F.) and by Bishop's University. The work of A.G. has been carried out in the framework of the activities of the Italian National Group for Mathematical Physics [Gruppo Nazionale per la Fisica Matematica (GNFM), Istituto Nazionale di Alta Matematica (INdAM)].
[1] B.P. Abbott et al. (LIGO Scientific Collaboration and Virgo Collaboration), Phys. Rev. Lett. 116, 061102 (2016).

[2] B.P. Abbott et al. (LIGO Scientific Collaboration and Virgo Collaboration), Phys. Rev. Lett. 116, 241103 (2016).

[3] LIGO Scientific Collaboration and Virgo Collaboration, Phys. Rev. X 6, 041015 (2016).

[4] B.P. Abbott et al. (LIGO Scientific and Virgo Collaboration), Phys. Rev. Lett. 118, 221101 (2017)

[5] E. Berti et al., Class. Quantum Grav. 32, 243001 (2015).

[6] T. Baker, D. Psaltis, and C. Skordis, Astrophys. J. 802, 63 (2015).

[7] S. Capozziello, S. Carloni, and A. Troisi, Rec. Res. Dev. Astron. Astrophys. 1, 625 (2003) arXiv:astro-ph/0303041; S.M. Carroll, V. Duvvuri, M. Trodden, and M.S. Turner, Phys. Rev. D 70, 043528
(2004).

[8] T.P. Sotiriou and V. Faraoni, Rev. Mod. Phys. 82, 451 (2010); A. De Felice and S. Tsujikawa, Living Rev. Relativity 13, 3 (2010); S. Nojiri and S.D. Odintsov, Phys. Repts. 505, 59 (2011).

[9] C.H. Brans and R.H. Dicke, Phys. Rev. 124, 925 (1961).

[10] P.G. Bergmann, Int. J. Theor. Phys. 1, 25 (1968); R.V. Wagoner, Phys. Rev. D 1, 3209 (1970); K. Nordvedt, Astrophys. J. 161, 1059 (1970).

[11] M.D. Roberts, J. Math. Phys. 37, 4557 (1996).

[12] M.D. Roberts, Gen. Rel. Grav. 21, 907 (1989).

[13] M.W. Choptuik, Phys. Rev. Lett. 70, 9 (1993).

[14] P.R. Brady, Class. Quantum Grav. 11, 1255 (1994).

[15] Y. Oshiro, K. Nakamura, and A. Tomimatsu, Prog. Theor. Phys. 91, 1265 (1994).

[16] V. Husain, E.A. Martinez, and D. Nuñez, Phys. Rev. D 50, 3783 (1994). 
[17] H.P. de Oliveira and E.S. Cheb-Terrab, Class. Quantum Grav. 13, 425 (1996).

[18] A. Wang and H. P. de Oliveira, Phys. Rev. D 56, 753 (1997).

[19] A.V. Frolov, Phys. Rev. D 61, 084006 (2000).

[20] H. Maeda, Phys. Rev. D 79, 024030 (2009).

[21] H. Maeda, Class. Quantum Grav. 32, 135025 (2015).

[22] R.M. de Almeida André, "Critical Behavior in Gravitational Collapse", MSc thesis, U. Lisbon, 2016.

[23] L.M. Burko, Gen. Relativ. Gravit. 29, 259 (1997).

[24] S.A. Hayward, Class. Quantum Grav. 17, 4021 (2000).

[25] G. Clement and S.A. Hayward, Class. Quantum Grav. 18, 4715 (2001).

[26] R.A. Sussman, J. Math. Phys. 32, 223 (1991).

[27] A.V. Frolov, Phys. Rev. D 56, 6433 (1997).

[28] A.V. Frolov, Phys. Rev. D 59, 104011 (1999).

[29] R.M. Wald, General Relativity (Chicago University Press, Chicago, 1984).

[30] I.I. Gutman and R.M. Bespalko, Sbornik Sovrem. Probl. Grav. Tbilisi 1, 201 (1967).

[31] C.W. Misner and D.H. Sharp, Phys. Rev. 136, B571 (1964); W.C. Hernandez and C.W. Misner, Astrophys. J. 143, 452 (1966).

[32] A. Giusti and V. Faraoni, Class. Quantum Grav. 37, 195005 (2020); V. Faraoni and A. Giusti, Symmetry 12, 1264 (2020).

[33] G.F. Bressange, Class. Quantum Grav. 15, 225 (1998).

[34] C. Barrabès and G.F. Bressange, Class. Quantum Grav. 14, 805 (1977).
[35] D.C. Robinson, Gen. Relativ. Gravit. 38, 153 (2006).

[36] R.H. Dicke, Phys. Rev. 125, 2163 (1962).

[37] J.P. Abreu, P. Crawford, and J.P. Mimoso, Class. Quantum Grav. 11, 1919 (1994); J. Sultana, Gen. Relat. Gravit. 47, 73 (2015).

[38] T. Clifton, D.F. Mota, and J.D. Barrow, Mon. Not. Roy. Astron. Soc. 358, 601 (2005).

[39] V. Faraoni, Phys. Lett. A 245, 26 (1998); Phys. Rev. D 59, 084021 (1999).

[40] J.L. Synge, Relativity: The General Theory (North Holland, Amsterdam, 1960).

[41] S.M. Carroll, Spacetime and Geometry: An Introduction to General Relativity (Addison Wesley, San Francisco, 2004).

[42] V. Faraoni, Cosmology in Scalar Tensor Gravity, Fundamental Theories of Physics Series vol. 139 (Kluwer Academic, Dordrecht, 2004).

[43] V. Faraoni, D.K. Çiftci, and S.D. Belknap-Keet, Phys. Rev. D 97, 064004 (2018).

[44] M.D. Roberts, Phys. Lett. B 795, 327 (2019).

[45] A. Banijamali, B. Fazlpour, and V. Faraoni, Phys. Rev. D 100, 064017 (2019).

[46] S.W. Hawking, Comm. Math. Phys. 25, 167 (1972); J.D. Bekenstein, arXiv:gr-qc/9605059 T.P. Sotiriou and V. Faraoni, Phys. Rev. Lett. 108, 081103 (2012); S. Bhattacharya, K.F. Dialektopoulos, A.E. Romano, and T.N. Tomaras, Phys. Rev. Lett. 115, 181104 (2015); V. Faraoni, Phys. Rev. D 95, 124013 (2017). 